\title{
Os caminhos da enfermagem: de Florence à globalização
}

Recebido em: 02/12/2010

Aceito em: 21/03/2011

Sonia Maria Rezende Camargo de Miranda ${ }^{2}$

Este artigo tem por objetivo mostrar a diversidade da atuação do enfermeiro no cenário da saúde nacional, desde o início da profissão até os tempos atuais. Reúne depoimentos e vivências de vários enfermeiros que atuam em diferentes segmentos. Com isso, podemos perceber a evolução do papel profissional cuja construção foi ancorada, em seu início, na religiosidade, obediência, submissão, ausência de autonomia, e não na especificidade de seu papel profissional, como se faz atualmente.

Descritores: Enfermagem, História da Enfermagem, Florence Nightingale, Globalização.

\section{The ways of nursing: from Florence up to globalization}

This article aims to show the diversity of nurses' performance in national health scene since the beginning of the occupation till today. It gathers testimonies and experiences of many nurses working in different segments. With this we can see the evolution of the professional role for which construction was rooted in its beginnings in religion, obedience, submissiveness, lack of autonomy, and non-specificity of their professional role as it does today.

Descriptors: Nursing, Nursing History, Florence Nightingale, Globalization.

\section{Los caminos de la enfermería: de Florence hasta la globalización}

Este artículo pretende mostrar la diversidad de los resultados de las enfermeras en la escena nacional de salud desde el inicio de la ocupación hasta la actualidad. Reúne testimonios y experiencias de muchas enfermeras que trabajan en diferentes segmentos. Con esto podemos observar la evolución de la función profesional para cuya construcción se basaba en sus inicios en la religión, la obediencia, la sumisión, la falta de autonomía, y la no especificidad de su función profesional, ya que en la actualidad.

Descriptores: Enfermería, Historia de la Enfermería, Florence Nightingale, Globalización.

\section{INTRODUÇÃO}

$\mathrm{P}$ ensando na transformação histórica dos modos de produção de saúde e na metamorfose que o mundo da enfermagem vem sofrendo, tivemos a ideia de fazer esta obra, que contemplasse a história da enfermagem, mas que alcançasse os distintos profissionais que atuam desde a assistência direta ao paciente até aqueles que ocupam cargos gerenciais e/ou desenvolvem atividades docentes (responsáveis pela formação de futuros enfermeiros). Gostaríamos que o estudo mostrasse a multiplicidade de atuação em diversos segmentos desse profissional no cenário da saúde. Nosso objetivo foi valorizar os bastidores da profissão e desmistificar o papel do enfermeiro, conhecido pela comunidade como aquele submisso e complementar ao papel médico. O objetivo é mostrar os avanços, as conquistas e a complexidade que envolvem os diferentes tipos do "cuidar", muitas vezes desconhecidos até pelos próprios pares, em relação à autonomia e abrangência das distintas maneiras de fazer enfermagem.

$\mathrm{Na}$ história da enfermagem, sua precursora no Brasil,
Anna Nery, nascida em 13 de dezembro de 1814, com 130 anos de passamento, que se completou em 20 de maio de 2010, já mostrava garra, competência e determinação, enfatizando a importância de lutar por maior independência no futuro. Participou da Guerra do Paraguai como voluntária e recebeu várias homenagens. Na época, não havia escolas de enfermagem no Brasil nem na Argentina, mas as pessoas que cuidavam eram chamadas de "enfermeiras", como é o caso de Anna Nery, considerada pela Sociedade Cruz Vermelha das Américas a pioneira da enfermagem no Brasil ${ }^{(1)}$.

Hoje, na profissão, são exigidas qualidade nos procedimentos técnicos e segurança para o paciente. Nas instituições, os enfermeiros devem ser multiqualificados e multifuncionais, pois desde Florence Nigtingale e Anna Nery prestam, na maioria das vezes, os cuidados mais complexos e administram os demais cuidados, por não existirem enfermeiros suficientes para "o cuidar direto".

No mundo, os enfermeiros devem dominar a linguagem da informática e das máquinas de alta tecnologia, possuir

1 Enfermeiro. Mestre em adminsitração, educação e comunicação pela Unimarcos. Coordenador da pós-graduação em saúde pública e PSF para enfermeiros - Universidade Gama Filho. Atua na Vigilância Sanitária da Secretaria Municipal de Saúde de São Paulo. Email: williammalagutti@hotmail.com

2 Enfermeira. Doutora em ciências sociais pela Pontifícia Universidade Católica de SP. Professora da Universidade Anhembi Morumbi e professora da pós-graduação da Universidade Gama Filho. 
raciocínio rápido e abstrato, ter iniciativa, ser criativos, competitivos, comunicativos, dominar idiomas, estar muito bem informados, além de possuir traços de líderes para formarem verdadeiros times. Para alcançar essa excelência, é necessária uma educação transdisciplinar em que se problematize a realidade e se desenvolva a criatividade para que o futuro profissional possa acompanhar as mudanças e estar conectado ao mundo global.

Dessa maneira, não basta apenas saber executar o cuidado. É fundamental hoje dominar o novo paradigma tecnológico, estar conectado com o mundo das informações e utilizar adequadamente ferramentas como o computador, para obter o máximo de vantagens do mesmo. Uma vez que o sistema de informação produz um grande volume de dados, cada vez mais se exige que o profissional seja capaz de receber, arquivar e distribuir informações de forma rápida e eficiente ${ }^{(2)}$. Isso é o que assistimos atualmente na profissão, bem diferente de outrora.

Segundo $\operatorname{Mott}^{(3)}$, a enfermagem no Brasil muito contribuiu na construção do perfil da profissão ao longo do século 20 e no século 21. Em artigo publicado na Revista Médica de São Paulo pelo doutor Moisés Amaral em 1906, referese ao profissional "enfermeiro" inapto e despreparado para preencher as exigências da medicina moderna. Infelizmente, "sem estudo, sem preparo e com uma prática muitas vezes rotineira, esses empregados não preenchem muitas vezes as exigências da medicina moderna". A falta de aptidão e a ignorância prejudicam os doentes e "esterilizam" os sacrifícios dos médicos(4).

No entanto, havia um dilema nessa época: preparar o profissional para torná-lo competente e ao mesmo tempo enfrentar o receio de que isso induzisse o enfermeiro a se tornar prepotente e pretender "a cura por sua conta"(4).

No Brasil, a mulher foi considerada a candidata nata ideal para a profissão, devido a suas habilidades e qualidades. Por outro

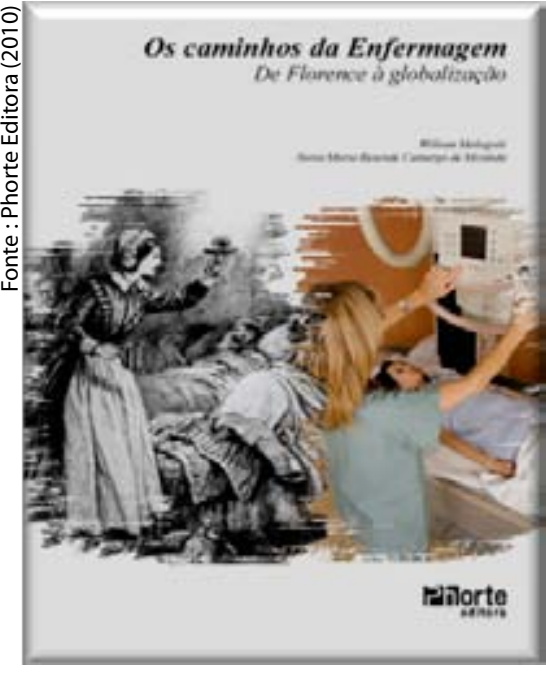

Figura 1. Os Caminhos da Enfermagem, de Florence à Globalização das pessoas. Dessa forma, abre possibilidades para um fazer compartilhado, no qual há lugar para a inventividade e criatividade no sentido ecológico do cuidar ${ }^{(5)}$.

O processo de enfermagem foi introduzido no Brasil por Wanda Horta na década de 1960, tornando-se uma metodologia que sistematizou as ações de enfermagem. Com a criação das taxonomias de enfermagem por volta dos anos 1970, teve-se como intenção inicial promover uma linguagem padronizada dos diagnósticos e prescrições de enfermagem entre esses profissionais. Essa, no entanto, não deixou de interferir no dilema da desumanização, pois passou a pensar o cuidado de maneira integral, universal e subjetivo. Entre as taxonomias, podemos citar: Nanda-I* (North American Nursing Diagnoses Association), dos Diagnósticos de Enfermagem, a Classificação dos Resultados de Enfermagem (NOC - Nursing Outcomes Classification) e a Classificação das Intervenções de Enfermagem (NIC - Nursing Interventions Classification). Essas taxonomias promovem uma linguagem unificada que permite ao enfermeiro planejar o cuidado com metas a serem alcançadas pela equipe ${ }^{(6)}$.

*A Nanda-I (Nanda International) é a nova razão social da antiga Nanda (North American Nursing Diagnoses Association), pelo fato de essa, atualmente, estar presente em diversos países, como Brasil, Equador, Peru, Venezuela, Japão, Espanha, Suíça e Alemanha, em grupos de trabalho que a configuram não mais como uma entidade americana, e sim internacional ${ }^{(6)}$.

Com esses instrumentos do exercício profissional, foi possível a implantação do Processo de Enfermagem, que se constitui nas fases: histórico, diagnóstico, prescrição e resultados obtidos, uma abordagem científica e sistemática que visa a planejar, implementar e avaliar intervenções de enfermagem. Apesar de não ser utilizado por todos os enfermeiros no Brasil, devido aos mais diversos fatores - que não iremos discutir no momento -, o processo de enfermangem é uma nas pesquisas sobre a história da enfermagem brasileira, para dirimir dúvidas quanto à distinção entre o papel do cuidar de médicos e enfermeiros, que não está bem definido nesse período, em que ambos os profissionais atuavam no cuidar e no curar ${ }^{(3)}$.

Ao tentarmos fazer uma prospecção de como será "o cuidar" no futuro, diante das contradições e da complexidade do mundo em que vivemos, nos deparamos com o antagonismo que enfrentamos atualmente na profissão: se de um lado há o desenvolvimento do modelo maquínico-tecnológico em favor do paciente, de outro esse dificulta as relações humanas, tornando-as racionais, frias e calculistas. Segundo Medeiros, há emergência de uma forma humanizante, centrada na alteridade e no alargamento do sentido dos "nós", no cuidar.

O SUS, em defesa da integralidade, um de seus princípios, propõe o diálogo, a recusa do reducionismo e da objetivação iniciativa da maior importância para tal.

\section{Diversidade do papel do enfermeiro no mundo globalizado}

Quando nos remetemos "ao cuidado", sempre nos vem à mente procedimentos técnicos de uma rotina fatigante hospitalar e/ou saúde coletiva. Entretanto, atualmente, o papel do enfermeiro é tão abrangente que nem os próprios enfermeiros têm conhecimento das diversas áreas de atuação de seus colegas. Somente na enfermagem do trabalho, por exemplo, Moraes $^{(7)}$ destaca sete desses como os mais importantes: o papel de gestor, pesquisador, consultor, docente e clínico/especialista, além de mediador entre os representantes das empresas, modificador da qualidade de vida dos trabalhadores. Mas a diversidade é ainda maior do que imaginamos. Elencaremos alguns tópicos que ilustram essa nova realidade. 


\section{Dor}

A dor é uma condição secular que acompanha as enfermidades. Nesse processo, o enfermeiro atua desde outrora convivendo com essa realidade. Antigamente, havia poucos analgésicos eficazes que aliviassem esse sofrimento. Atualmente, além da disponibilidade no mercado de analgésicos potentes, a enfermagem utiliza a SAE - Sistematização da Assistência de Enfermagem, com o objetivo de aliviar o sofrimento, contribuindo para a integridade global do corpo e do paciente com dor.

No processo de enfermagem, a entrevista e o exame físico fornecem dados significativos para identificar a etiologia da dor e conhecer o comportamento doloroso dos doente ${ }^{(6)}$. As informações devem ser colhidas dos pacientes e de seus cuidadores. Na investigação, são coletados dados objetivos (sinais) e subjetivos (sintomas), que influenciam a percepção e a resposta a dor.

O reconhecimento pelos enfermeiros de um método de trabalho sistemático, por meio da Sistematização da Assistência de Enfermagem ( $\mathrm{SAE}$ ), garante visibilidade e reconhecimento profissional, além de permitir uma avaliação da prática ${ }^{(8,9)}$.

\section{Gestantes com tuberculose}

A tuberculose é uma doença reemergente em tempos atuais, devido à infecção pelo HIV. Seu tratamento e cura constituem um grande desafio para a equipe de enfermagem e para os pacientes. No caso específico de gestantes, o diagnóstico da patologia pode ser dificultado pelos sintomas inespecíficos aumento da frequência respiratória, fadiga e mal-estar -, que podem ser confundidos com demais queixas das gestantes.

É importante para o enfermeiro atuante nessa condição não só atuar junto às gestante como também a puérperas e neonatos, realizando o diagnóstico precoce, a terapêutica adequada e a vigilância epidemiológica da doença, do doente, da família e comunidade. Tudo isso pode ser feito de maneira mais eficaz através do suporte da Estratégia da Saúde da Família (ESF).

\section{Transplante de tecidos musculoesqueléticos}

Uma área pouco conhecida de atuação do enfermeiro é a captação de tecidos musculoesqueléticos de doadores falecidos - aloenxerto -, que contribui para a qualidade de vida de pacientes que necessitam desse procedimento. $O$ plano de captação é programado previamente para o caso dos tecidos a serem retirados nas cirurgias ortopédicas e odontológicas.

A equipe de enfermagem, composta de enfermeiros especializados, é supervisionada a distância por um médico ortopedista da área. Essa realiza todo o procedimento cirúrgico relacionado com a captação dos principais tecidos disponibilizados pelo banco, tais como: tálus, cristas ilíacas, fêmures, tíbias, fíbulas, calcâneos, tendões tibiais, patelares, extensores e Aquiles, entre outros. Esse processo é realizado em condições assépticas, como numa cirurgia ${ }^{(10)}$.

\section{Captação de tecidos oculares}

Outro procedimento realizado pelo enfermeiro é a atividade de enucleação, que está embasada no parecer n 4430/99 do
Conselho Regional de Medicina de São Paulo (Cremesp). Esse esclarece que "a atividade de enucleação pelo profissional enfermeiro entende-se condicionada ao adequado e necessário treino prévio. Outrossim, subordina-se a toda legislação vigente, particularmente as normas específicas para o funcionamento dos Bancos de Olhos e a Lei de Transplantes".

\section{Liderança Coaching}

Para Araújo Coaching, a liderança comprometida enxerga a pessoa como um todo, com seu desenvolvimento e sua realização para que possa enfrentar com maior agilidade as organizações ${ }^{(11)}$.

Essa ferramenta é adotada por profissionais de enfermagem em alguns hospitais do Brasil, com propostas de melhorar a qualidade assistencial dos pacientes, e tem sido um método eficiente, com resultados comprovados, embora não seja o único como referência no exercício da liderança do enfermeiro ${ }^{(11)}$.

A liderança situacional tem cada vez mais sido usada pelos gerentes de enfermagem. Nesse tipo de liderança, o gestor leva em consideração a maturidade de seus colaboradores. Quanto mais maduro o colaborador, menor o uso da autoridade, pois ele decide qual o tipo de comportamento em relação à tarefa.

\section{Gerenciamento de riscos}

Os enfermeiros são muitas vezes responsáveis pela avaliação de serviços de saúde. Utilizam como ferramentas a gestão de riscos, pois estão aptos a realizar o diagnóstico institucional e propor intervenções com a finalidade de prevenção de riscos e danos e aumentar a qualidade da assistência, voltados para a segurança do paciente. Também tem como objetivo preservar o estado patrimonial, financeiro, ético-moral e profissional dentro dos estabelecimentos de saúde ${ }^{(11,12)}$.

A auditoria de risco é um novo campo de atuação para o enfermeiro brasileiro inserido na área da administração dos serviços de saúde e gerenciamento de enfermagem ${ }^{(13)}$.

\section{latrogenias - segurança do paciente/suporte ao paciente crítico}

latro - prefixo de origem grega, indicando uma relação com o médico e com a medicina; iatrogênico - diz-se de um distúrbio ou doença provocado pelo médico ou equipe de enfermagem durante a prestação da assistência.

Geralmente os fatores relacionados às iatrogenias são encontrados em pacientes internados em Unidades de Tratamento Crítico, extremamente complexos e dependentes da equipe, recebendo duas vezes mais drogas, com múltiplas interações, o que aumenta a exposição dos profissionais ao erro.

Além disso, podemos elencar outros fatores, como: características que influenciam a equipe de trabalho; falta de competência individual; motivação; saúde física e mental alteradas; desatenção; fadiga. Um fator que é de extrema importância são os ruídos da comunicação entre equipes e seus pares, bem como a falta de educação permanente.

No ambiente de trabalho, podemos perceber alguns itens: sobrecarga laboral, manuseio de equipamentos, ambiente físico, exposição a agentes físicos, químicos e biológicos. 
Uma das iatrogenias mais discutidas entre os intensivistas é a utilização da tecnologia nas Unidades de Terapias Intensivas, que pode dificultar a relação profissional/paciente. Em 1999, a Terapia Intensiva brasileira desenvolveu o QUATi - Sistema de Informação em Pacientes Críticos, um instrumento de indicadores de gestão e de risco, capaz de mensurar e comparar o desempenho assistencial.

Esse sistema possui um banco de dados (são dados com índice de gravidade que permitem a descrição quantitativa do grau de disfunção orgânica do paciente crítico em valor numérico), cujos indicadores norteiam o funcionamento da UTI.

\section{Gerenciamento de paciente infartado - Case Managemant}

OGerenciamento de Casos (GC), termo originário da língua inglesa como Case Management, é uma série de processos de seleção e gerenciamento da assistência prestada a pacientes portadores de patologias específicas, visando a garantir a qualidade e a continuidade do atendimento prestado e a minimizar custos. $\mathrm{O}$ objetivo principal do GC é a redução de custos desnecessários por meio da otimização do tratamento ${ }^{(14)}$.

O gerenciamento dos casos é realizado pelo case manager (gerenciador de casos), que pode ser um enfermeiro, um farmacêutico ou biomédico, seguindo um protocolo assistencial que sofre modificações de acordo com as áreas assistenciais envolvidas no atendimento ao paciente.

No estudo Case Management Caseload Data(15), do qual participaram 522 enfermeiros gerenciadores de casos, observou-se que $36,5 \%$ desses profissionais atuavam em hospitais voltados ao atendimento de casos agudos e que $78 \%$ deles possuíam no mínimo dez anos de experiência assistencial antes se tornarem gerenciadores de casos.

\section{Gerenciamento de enfermagem em instituição pública}

Com o advento do SUS, algumas instituições públicas de saúde estão adotando como referencial um modelo de gestão com planejamento estratégico que contemple o sistema hierarquizado com uma sistematização administrativo-assistencial que se compõe de: comissões internas de trabalho, comissões científicas, comissão de ética em enfermagem, auditoria de prontuários, relatório gerencial e indicadores de absenteísmo.

Essa sistematização permite ao gestor uma visão ampliada do serviço de enfermagem em suas especificidades e corrobora para diminuir eventos adversos e iatrogenias.

\section{CONSIDERAÇÕES FINAIS}

Há uma diversidade ampla de atuação de profissionais enfermeiros no cenário de saúde nacional, que foi discutida em nosso livro, e sobre a qual não poderemos discorrer neste artigo, face à amplitude e abrangência de papéis profissionais que podem ser desempenhados pelos enfermeiros, tais como: o educador na formação e desenvolvimento de profissionais na área de oncologia e outras; hotelaria hospitalar; remoção aeromédica; terapias complementares no cuidar; promoção de saúde em povos indígenas; saúde ambiental; o enfermeiro como representante de vendas; auditoria, líder político e sindical.
Ao assistir ao paciente, gerenciar equipes, formar, capacitar profissionais de enfermagem, realizar pesquisas e demais atividades de competência dos enfermeiros, há necessidade dos mesmos estarem embasados sempre em princípios éticos, refletindo sobre seu fazer e agir, de acordo com as transformações do mundo globalizado.

\section{Referências}

1. Porto, F, Ott TR. Tributo a Anna Justina Ferreira Nery - pelos 130 anos do passamento. In: Malagutti W, Miranda SMRC. Os caminhos da enfermagem, de Florence à globalização. São Paulo: Phorte; 2010.

2. Nichiata LYI, Takahashi TF, Fracolli LA, Gryschek ALFPL. Relato de uma experiência de ensino de enfermagem em saúde coletiva: a informática no ensino de vigilância epidemiológica. Rev Esc Enferm USP. 2003.

3. Mott ML. Revendo a história de enfermagem no Estado de São Paulo 1890-1920. In: Malagutti W, Miranda SMRC. Os caminhos da enfermagem, de Florence à globalização. São Paulo: Phorte; 2010.

4. Notícias. Revista Médica de São Paulo. 1909;12(6):107.

5. Germano RG. A enfermagem do passado e do futuro: perspectivas e desafios do cuidar. In: Malagutti W, Miranda SMRC. Os caminhos da enfermagem, de Florence à globalização. São Paulo: Phorte; 2010. 6. North American Nursing Association. Diagnósticos de enfermagem da Nanda: definiçōes e classificação - 2007-2008. Porto Alegre: Artmed; 2008. 7. Morais AM. Aspectos históricos, conceituais e funcionais da enfermagem do trabalho e sua relação com saúde ocupacional. In: Malagutti W, Miranda SMRC. Os caminhos da enfermagem, de Florence à globalização. São Paulo: Phorte; 2010.

8. Iyer PW, Taptich BJ, Bernocchi-Losey D. Processo e diagnóstico de

enfermagem. Porto Alegre: Artes Médicas; 1993.

9. Garcia TR, Nóbrega MML. Processo de enfermagem e os sistemas de classificação dos elementos da prática profissional: instrumentos metodológicos e tecnológicos do cuidar. In: Santos I. São Paulo: Atheneu; 2004. p. 37-63.

10. Santos LAU. Assistência de enfermagem nos transplantes de tecidos musculoesqueléticos: da captação ao uso clínico. In: Malagutti W, Miranda SMRC. Os caminhos da enfermagem, de Florence à globalização. São Paulo: Phorte; 2010.

11. Araújo A. COAH: um parceiro para seu sucesso. São Paulo: Ed. Gente; 1999.

12. Feldman LB, organizadora. Gestão de risco e segurança hospitalar. Prevenção de danos ao paciente, notificação, auditoria de risco, aplicabilidade de ferramentas, monitoramento. São Paulo: Martinari; 2008. p. 376.

13. Feldaman LB. Gestão de riscos: novo campo de atuação para o enfermeiro. In: Malagutti W, Miranda SMRC. Os caminhos da enfermagem, de Florence à globalização. São Paulo: Phorte; 2010.

14. Case Management Caseload Data. Results of a national survey. [Internet]. [citado em 2005 Mai 28]. Disponivel em: http://www.ahcpub.com/ ahc_root_html/hot/archive/caseload_summary.pdf

15. Makdisse M, Santos BFC, Fernandes Jr CJ, Cendoroglo Neto M. Gerenciamento de doenças e casos em terapia intensiva. In: Knobel E. Condutas no paciente grave. 3a ed. São Paulo: Atheneu; 2006. p. 1999-2005. 